\title{
KINETICS OF BIODEGRADATION OF PHENOL AND A POLYPHENOLIC COMPOUND BY A MIXED CULTURE CONTAINING PSEUDOMONAS AERUGINOSA AND BACILLUS SUBTILIS
}

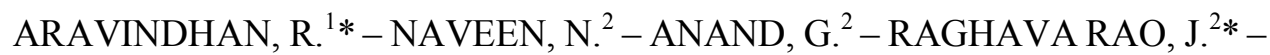 \\ UNNI NAIR, B. ${ }^{2}$ \\ ${ }^{1}$ Leather Process Technology Division, CSIR-Central Leather Research Institute \\ ${ }^{2}$ Chemical Laboratory, CSIR-Central Leather Research Institute \\ Adyar, Chennai- 600020 \\ (phone: +91442441 1630; fax: +91442491 1589) \\ * Corresponding authors \\ e-mail: aravindhan@clri.res.in; rao_clri@yahoo.com \\ (Received $20^{\text {th }}$ Nov 2007; accepted $22^{\text {nd }}$ July 2014)
}

\begin{abstract}
Biological degradation of phenolic compounds by a mixed bacterial culture was investigated. Pseudomonas aeruginosa and Bacillus subtilis were the two strains used in the study. $\mathrm{pH}$ and the temperature for the growth of bacteria was found to be $7 \pm 0.2$ and $37 \pm 2^{\circ} \mathrm{C}$, respectively. The mixed microbial culture was able to degrade $250 \mathrm{mgl}^{-1}$ of phenol and wattle completely within 36 and 48 hours, respectively. The growth kinetics of the mixed culture was also investigated over a wide range of initial concentrations of phenol and wattle by using Haldane's model. The Haldane growth kinetic model adequately described the degradation of phenolic compounds by the mixed microbial culture. Both phenol and wattle were found to inhibitory compounds. The decay coefficient for the growth of microbial culture on phenol and wattle was found to be 0.0069 and $0.0082 \mathrm{~h}^{-1}$, respectively.
\end{abstract}

Keywords: phenol, wattle, synergism, biodegradation, growth kinetics

\section{Introduction}

Due to their widespread use and subsequent introduction into the environment, phenolic compounds have been included to the list of pollutants by the environmental protection agencies. When discharged, phenolic wastes impart a carbolic odour to the river and are toxic to aquatic animals (Dean-Ross, 1995). The toxicity of phenol and phenolic compounds often result in the reduction of bio-treatment efficiency of wastewaters even at relatively low concentrations (Ahmed, 2001). Although physiochemical methods have been employed for removal of phenol and its derivatives, biological methods are preferred as the former is costly and often produce other undesirable products which are toxic, requiring further processing steps.

Treatment of phenolic wastes can be done using microbes or chemicals or by physical means. Bacteria play a major role in the degradation of phenol in soil, sediment and water. Using bacteria to digest waste and environmentally harmful compounds has several advantages over conventional treatment systems (Ojumu, 2005). The cost of remediation is less than that for conventional treatments. The diversity of microbes available makes the treatment of a wide variety of waste possible. Bacterial degradation of compounds is usually complete under appropriate conditions; toxic compounds are often completely converted into harmless compounds. Benzoate, catechol, cis-cis- 
muconate, $\beta$-ketoadipate, succinate and acetate have all been identified as intermediates in the biodegradation of phenol (Baradarajan, 1995).

For the cleanup to be achieved within a reasonable time frame without the threat of persistent, undesirable by-products, the entire system must be optimized. The need for optimization stems from the fact that the effect of microbes acting together is even better than their individual effects. This phenomenon is termed as 'synergism' (Zache, 1988). Biological degradation of organic substrates using pure microbial cultures occasionally produce toxic intermediates, which can be overcome by the use of mixed cultures that have a wider spectrum of metabolic properties.

In order to describe the substrate biodegradation, it is essential to evaluate the relationship between the specific growth rate and the initial substrate concentration. The bacteria behave differently when exposed to different initial substrate concentration and other experimental conditions. Hence, knowledge on both the rate and the extent of degradation is essential for improvements in the process control and phenol removal efficiency. In the present study, the biodegradation of phenolic compounds in the mixed microbial consortia containing $P$. aeruginosa and $B$. subtilis has been demonstrated and the bio-kinetic parameters have been evaluated.

\section{Materials and methods}

Spray dried wattle extract of commercial grade was used. All other chemicals used were of analytical and biological grade.

\section{Source of bacteria}

The Pseudomonas bacteria were isolated from the soil near the wastewater treatment plant at the premises of Central Leather Research Institute, Chennai, India. The Bacillus subtilis strain was obtained from the Microbiology Laboratory, Central Leather Research Institute, Chennai, India.

\section{Estimation of phenol}

Modified Folin-Ciocalteu's method (Clesceri, 1998) was employed for the estimation of phenol. Phenol solution of various concentrations was prepared by adding known volumes of phenol from the stock to the media. $1 \mathrm{ml}$ of phenol solution of various concentrations was added to the labeled test tubes to which, $0.5 \mathrm{ml}$ of media was serially added followed by $3.5 \mathrm{ml}$ of sodium carbonate and $5 \mathrm{ml}$ of de-ionized water. Finally $1 \mathrm{ml}$ of $1 \mathrm{~N}$ Folins Ciocalteu reagent was added and the absorbance was measured after a period of 25 minutes. Absorbance was measured at $760 \mathrm{~nm}$ in a 35 Lambda UV-visible spectrophotometer. A standard graph was plotted using the absorbance values obtained against corresponding concentrations of phenol. The blank used had the same composition of reagents except that $1 \mathrm{ml}$ of phenol solution added was replaced by $1 \mathrm{ml}$ of water.

\section{Isolation of the microbe}

The isolation of microbes was carried out by inoculating the microbes from the nutrient broth into the selective medium using a sterile inoculation loop in a laminar airflow chamber. The selective medium favors the growth of only a particular microbe and inhibits the growth of others. For the isolation of Pseudomonas sp. Pseudomonas

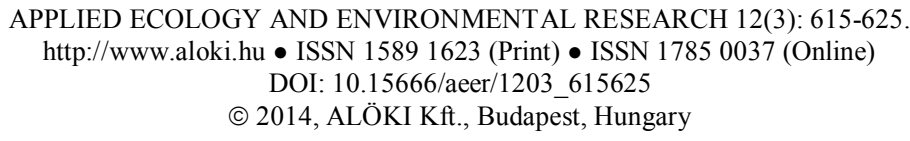


selective medium procured from Qualigens Fine Chemicals was employed. The medium after sterilization was poured in the petri plates or test tubes and left for solidification. After solidification of the medium, the bacterial samples were inoculated in the petri plates or test tubes.

\section{Identification of species}

The bacterial species identified were subjected to biochemical analysis in order to ascertain the genus of the species. Various biochemical tests like catalase test, citrate utilization test, starch hydrolysis, methyl red test, Voges Proskauer test were carried out as per standard procedures (Cappuccino, 1996). The optimum growth conditions like $\mathrm{pH}$ and temperature were also determined.

\section{Effect of $\mathrm{pH}$}

$\mathrm{pH}$ of the medium is a very important factor, which influences the growth of microorganisms. The inoculation medium was prepared by adjusting it to required $\mathrm{pH}$ values (4 to 11) by employing either $0.1 \mathrm{~N} \mathrm{NaOH}$ or $0.1 \mathrm{~N} \mathrm{HCl}$. Optical density $\left(\mathrm{OD}_{400 \mathrm{~nm}}\right)$ of the inoculated medium was measured at $0^{\text {th }}$ hour soon after the inoculation of microbe and was again measured after 24 hours to estimate the growth of microbes at respective $\mathrm{pH}$ 's.

\section{Synergistic degradation of phenol and wattle by P. aeruginosa and B. Subtilis}

B. subtilis and P. aeruginosa were used as mixed culture in the degradation of phenol and wattle. The use of selective media was not recommended in this operation because the chemical present in one selective medium may inhibit the action of the other microorganism. Hence nutrient broth was used for the purpose of mixed degradation. Hence, extreme care was taken during inoculation and incubation as risk of using nutrient broth is very large. To the nutrient broth, loop full of $P$. aeruginosa and $B$. subtilis were inoculated and incubated at $37 \pm 1{ }^{\circ} \mathrm{C}$ in an incubator shaker. Once an initial $\mathrm{OD}_{400}$ of $0.8 \pm 0.2$ was attained, known quantity of phenol and wattle from the stock solution was added to nutrient broth and made up to known volume, respectively and kept in an incubator shaker. The $\mathrm{OD}_{400}$ was monitored at regular intervals to monitor the growth of microbes. The change in substrate concentrations with time was also estimated by modified Folin's method. The growth as well as the change in the concentration of phenol was monitored using Lambda $35 \mathrm{UV}-\mathrm{V}$ is spectrophotometer. The substrates concentration in the medium was calculated from the standard graph for the particular substrate.

\section{Results and Discussions}

\section{Growth of microbes in the selective medium}

The nutrient broth prepared and inoculated with the microbes showed immense turbidity after 24 hours of incubation. The microbial samples from the nutrient broth were grown in a Pseudomonas selective medium. In order to cultivate the individual species of Pseudomonas, the consortia were inoculated in 4 different selective media ( $P$. aeruginosa, $P$. putida, $P$. cepacia, $P$. fluroscens). The abundant growth was observed in the $P$. aeruginosa selective medium. This proves $P$. aeruginosa is the dominant species 
present in the soil. All the other microbes were either completely absent or must be present in trace quantities.

\section{Biochemical characterization}

Biochemical characterization has been carried out in order to ensure the presence of the microbe of our interest. The results for the biochemical characterization of the bacterial species are shown in (Table 1). The formation of effervescence has been observed in the test tube inoculated with $P$. aeruginosa and B. subtilis, which indicates a catalase activity. Simmons citrate test has been positive in case of Pseudomonas and $B$. subtilis (appearance of blue color). Pseudomonas answered negative starch test, where as $B$. subtilis showed a visible color change. The formation of red ring has been observed with B. subtilis, whereas no ring was observed in Pseudomonas, which indicated a negative methyl red test. From the table, it is clear that the microbe employed are $P$. aeruginosa and B. subtilis.

Table 1. Biochemical characterization of the microbes

\begin{tabular}{|c|c|c|}
\hline Test performed & P. aeruginosa & B.subtilis \\
\hline Catalase test & + & + \\
Simmon citrate test & + & + \\
Starch test & - & + \\
Methyl red test & - & + \\
VP test & + & + \\
\hline
\end{tabular}

\section{Effect of $\mathrm{pH}$ and temperature}

To find out the favourable $\mathrm{pH}$ for the growth of $P$. aeruginosa and B. subtilis, the growth profile of the microbes have been observed over time by monitoring the change in $\mathrm{OD}_{400 \mathrm{~nm}}$ at different $\mathrm{pH}$ values (Fig. 1). Figure clearly shows that P. aeruginosa and $B$. subtilis had maximum growth at $\mathrm{pH}$ of $7 \pm 1$. Hence, for subsequent experiments the $\mathrm{pH}$ of the nutrient broth has been maintained at $7 \pm 0.2$.

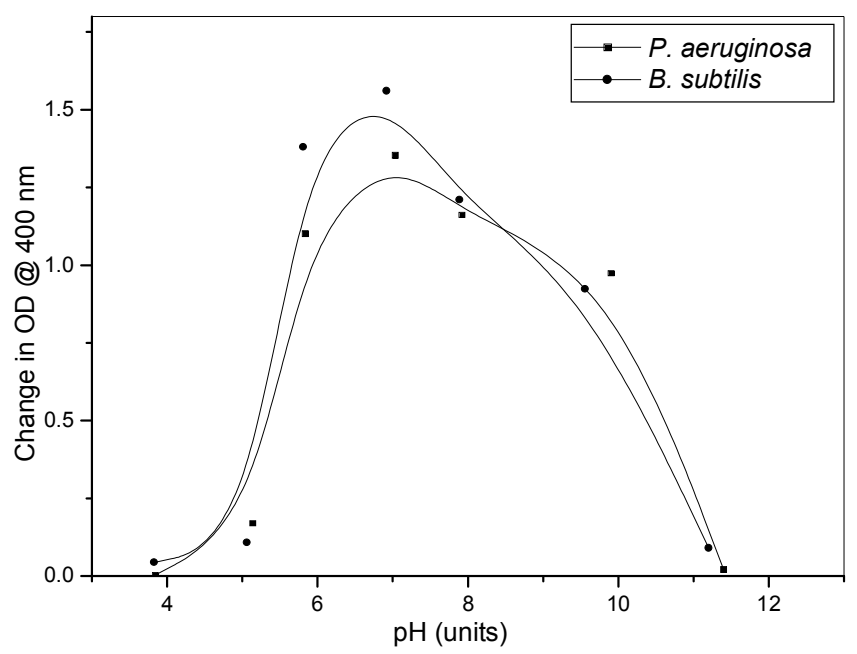

Figure 1. Effect of $p H$ on the growth of P. aeruginosa and B. subtilis

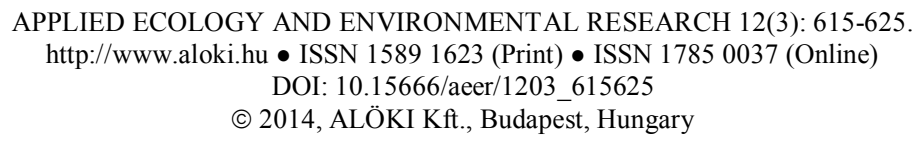


Once the $\mathrm{pH}$ has been optimized, the effect of temperature on the growth of microbes has been studied. The optimal temperature for the growth of these microbes is determined to be around $37 \pm 2 \mathrm{oC}$. Hence, the subsequent experiments have been carried out at this temperature.

\section{Effect of initial substrate concentration}

Spray dried wattle extract, a polyphenolic material, used as a tanning and retanning agent during leather manufacture was selected as a representative substrate for phenol. In order to find out the maximum concentration with which the mixed culture of $P$. aeruginosa and $B$. subtilis can grow, the microbes were grown in the presence of different initial concentrations of phenol and wattle. The initial concentrations of phenol and wattle were varied from 25, 50, 100, 250 and $500 \mathrm{mgl}^{-1}$. The degradation profile of phenol and wattle were monitored at regular intervals using modified Folin's method. The growth of microbes was monitored over time. (Figs. 2 and 3) provide the phenol and wattle degradation data at an initial concentration of $250 \mathrm{mgl}^{-1}$ of the substrate. The measurement of growth of microbe and the residual concentration of the substrate were followed till the substrate present got completely consumed. The results showed that the higher the concentration of the substrates higher the time taken to consume it fully. From the figures, it is very clear that the mixed microbial culture containing $P$. aeruginosa and $B$. subtilis was able to consume phenol and wattle completely. The consumption of $250 \mathrm{mgl}^{-1}$ of phenol took 36 hours, while the consumption of $250 \mathrm{mgl}^{-1}$ of wattle took almost 48 hours. Wattle being a polyphenolic compound took a longer time for degradation when compared to phenol alone.

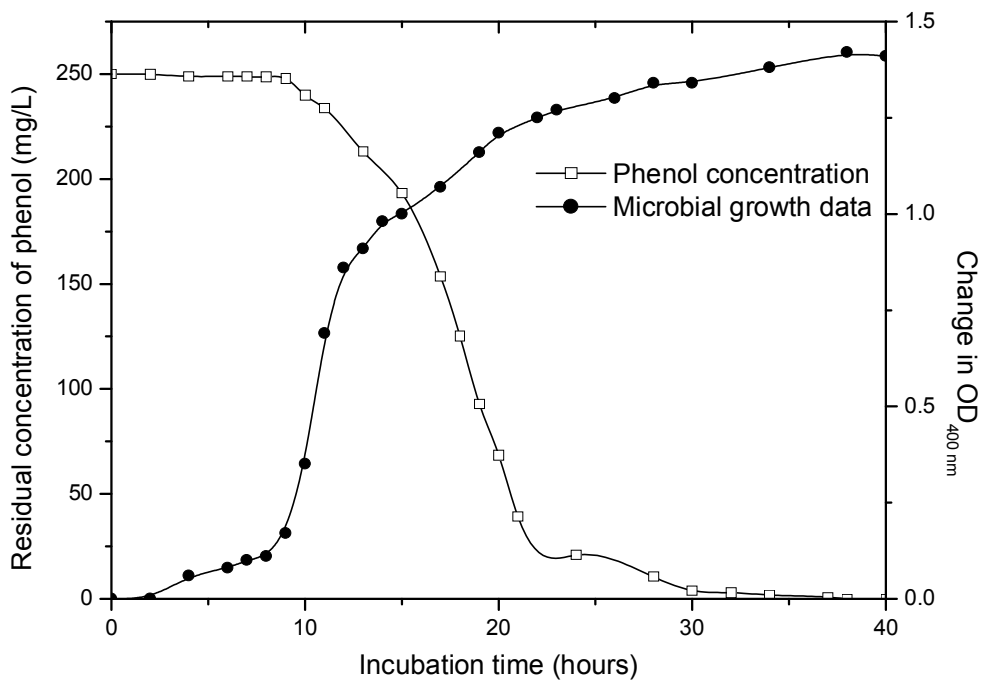

Figure 2. Degradation of phenol by the mixed microbial culture (Initial conc $=250 \mathrm{mgl}^{-1} ; \mathrm{pH}=$ 7.0 $\pm 0.2 ;$ Temp $=37 \pm 2{ }^{\circ} \mathrm{C}$ )

\section{Growth kinetics}

The growth kinetics of the mixed culture has been evaluated from the microbial growth data at different initial phenol and wattle concentration. The plot of OD against

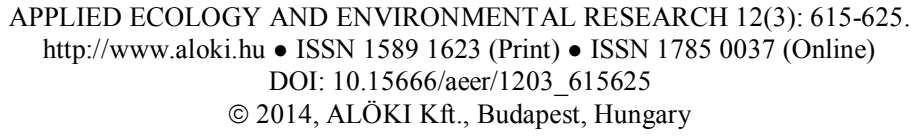


the initial phenol concentration has been used for the determination of specific growth rate (Livingston, 1989). The specific growth rate has been determined from the slope of the linear plots of OD vs Time during the exponential phase at respective initial phenol concentration.

Cell growth kinetics in a batch reactor may be modelled by the following equation (Edwards, 1970):

$$
\frac{d X}{d T}=\mu_{g} X-k_{d} X=\mu_{n e t} X
$$

For substrate:

$$
\frac{d S}{d t}=-\frac{1}{Y}\left(\frac{d X}{d t}\right)
$$

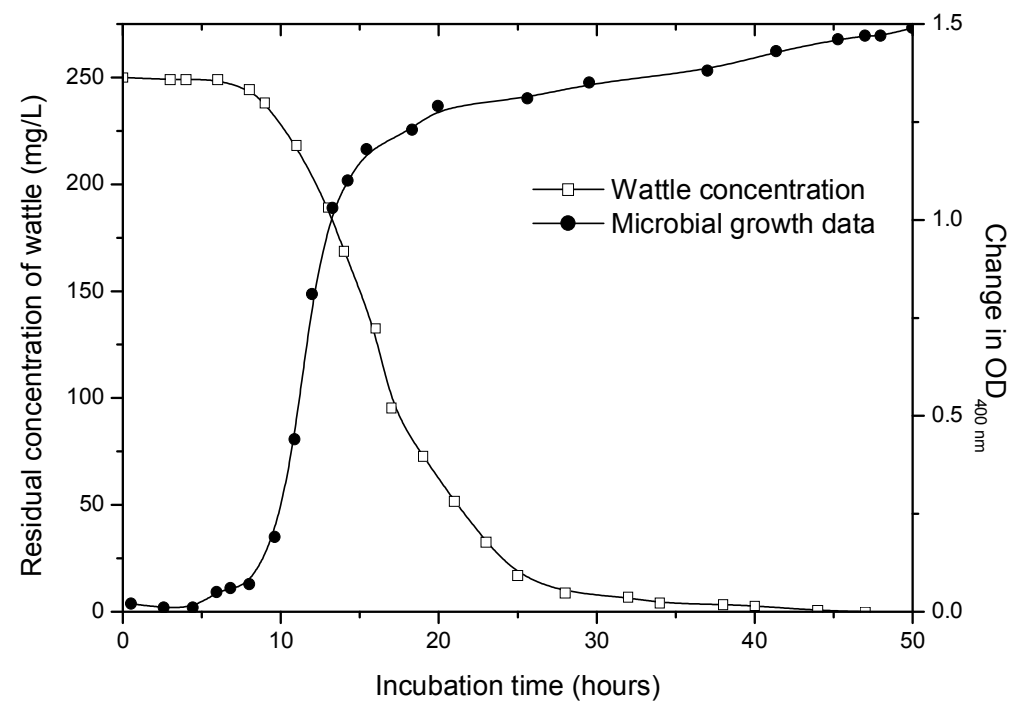

Figure 3. Degradation of wattle by the mixed microbial culture (Initial conc $=250 \mathrm{mgl}^{-1} ; \mathrm{pH}=$ 7.0 $\pm 0.2 ;$ Temp $=37 \pm 2{ }^{\circ} \mathrm{C}$ )

The specific growth rate $(\mu \mathrm{g})$ is a function of substrate concentration $(\mathrm{S})$. Monod and Haldane kinetics are the two most widely used equations to calculate the growth kinetics of any microbial systems. According to Monod's equation, the substrate is considered a non-inhibitory compound and the Monod's non inhibitory kinetics equation is given as below:

$$
\mu_{g}=\frac{\mu_{\max } S}{K_{s}+S}
$$


Whereas, the Haldane's kinetic equation considers the substrate to an inhibitory compound. Haldane's growth model was selected due to its mathematical simplicity and wide acceptance for representing the growth kinetics of inhibitory substrates. The Haldane's inhibitory growth kinetics equation is as follows:

$$
\mu_{g}=\frac{\mu_{\max } S}{K_{s}+S+\left(S^{2 /} K_{i}\right)}
$$

At higher substrate concentrations, $\mathrm{S}>>\mathrm{Ks}$, the above equation reduces to the following:

or

$$
\mu_{g}=\frac{\mu_{\max } S}{S+\left(S^{2} / K_{i}\right)}
$$

$$
\frac{1}{\mu_{g}}=\frac{1}{\mu_{\max }}+\frac{S}{\left(K_{i} \mu_{\max }\right)}
$$

This is the linearized Haldane's equation.

The experimental $\mu$ data obtained have been plotted against the respective initial concentration of phenol and wattle, respectively. (Figs. 4 and 5) show a typical trend that the values of $\mu$ increases with increase in initial substrate concentration up to certain initial concentration of the substrates and then it starts decreasing with further increase in initial concentration. This observation infers that both phenol and wattle are inhibitory types of substrates. Thus the Haldane's growth kinetic model has been used, which represents growth kinetic data of an inhibitory compound.

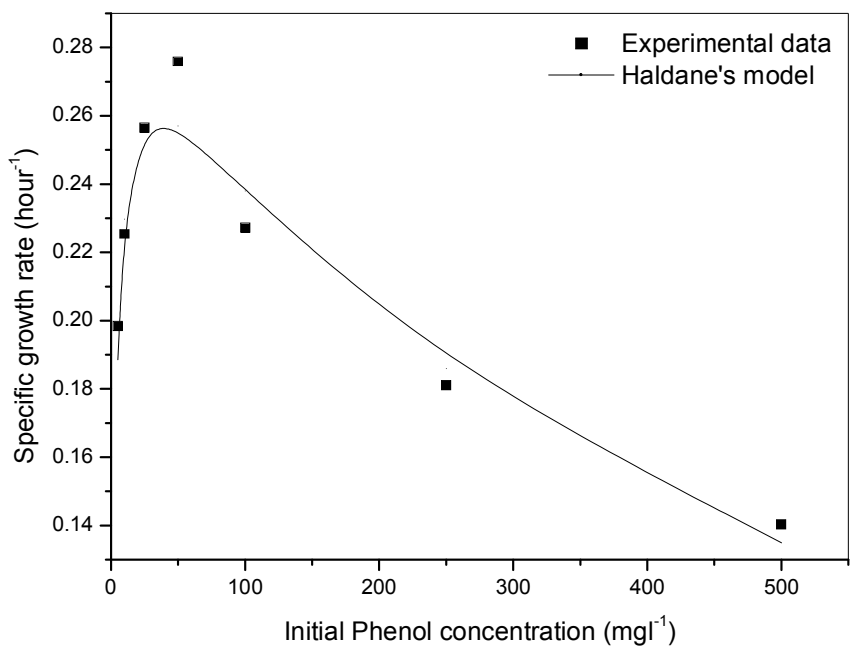

Figure 4. Haldane's growth model fitted to the degradation of phenol by the mixed microbial consortia to determine the growth kinetic parameters. 


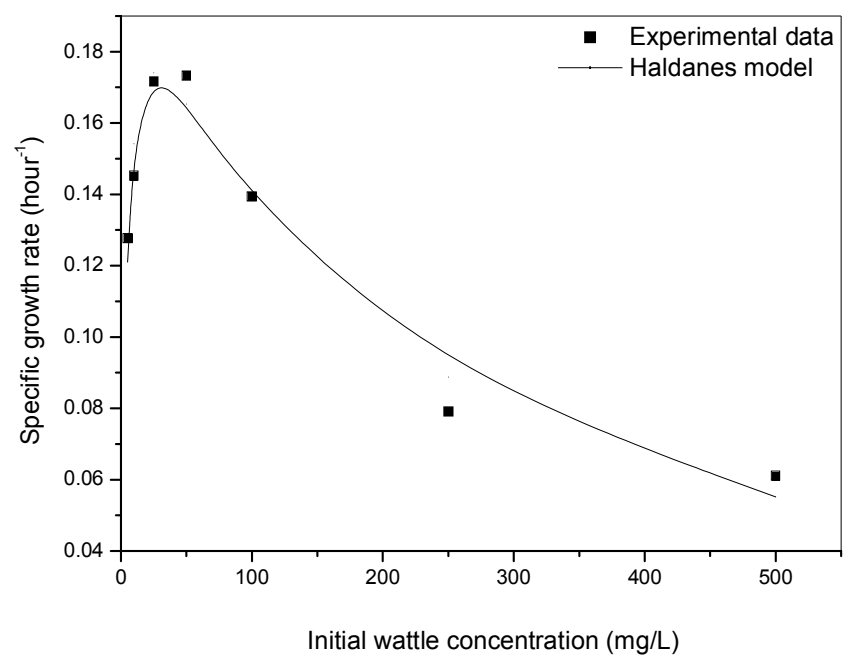

Figure 5. Haldane's growth model fitted to the degradation of wattle by the mixed microbial consortia to determine the growth kinetic parameters.

The estimation of the parameters using Linearized Haldane's model have been calculated and provided in (Table 2). Nonlinear regression technique has been adopted for the estimation of parameters of Haldane model. The experimental data have been solved for the Haldane equation using SPSS 15 for Windows, statistical software. The values for the linearized Haldane equation have been used as the starting guess values for the nonlinear regression fits. It could be observed from the figures that Haldane's model represents well the growth kinetics of the mixed culture. Also, the $\mathrm{R}^{2}$ value has been observed to be above 0.930 in both the cases. The calculated parameter values are provided in (Table 2). The values of maximum specific growth rate $\mu_{\max }(0.305$ and $0.233 \mathrm{~h}^{-1}$ for phenol and wattle) and the substrate inhibition coefficient $\mathrm{K}_{\mathrm{i}}$ (399 and 155 $\mathrm{mgl}^{-1}$ for phenol and wattle), were comparable to the values already published in various literatures (Pawlowsky, 1973 and Kumar, 2005). The values of substrate half saturation coefficient $\mathrm{K}_{\mathrm{s}}$ was also well within the range (3.02 and $4.48 \mathrm{mgl}^{-1}$ ) (Kumaran, 1997).

Table 2. Growth kinetics parameter values of linearized (LHM) and non-linearized (NLHM) Haldane's model for biodegradation of phenol and wattle by the mixed consortia of $P$. aeruginosa and B. subtilis

\begin{tabular}{|c|c|c|c|}
\hline \multicolumn{2}{|c|}{ Parameters } & Phenol & Wattle \\
\hline \multirow{3}{*}{ LHM } & $\mu_{\max }\left(\mathrm{h}^{-1}\right)$ & 0.244 & 0.164 \\
& $\mathrm{~K}_{\mathrm{i}}\left(\mathrm{mgl}^{-1}\right)$ & 702 & 289 \\
& $\mathrm{R}^{2}$ & 0.800 & 0.915 \\
& $\mu_{\max }\left(\mathrm{h}^{-1}\right)$ & 0.305 & 0.233 \\
NLHM & $\mathrm{K}_{\mathrm{i}}\left(\mathrm{mgl}^{-1}\right)$ & 399 & 155 \\
& $\mathrm{~K}_{\mathrm{s}}\left(\mathrm{mgl}^{-1}\right)$ & 3.02 & 4.48 \\
& $\mathrm{R}^{2}$ & 0.935 & 0.971 \\
\hline
\end{tabular}




\section{Endogenous or decay coefficient}

A typical growth curve shows a decline in cell population after the complete consumption of substrate. During this declining phase some part of the cell population becomes the food for the rest of the cell population. This part of the growth curve in a batch reactor has been modelled by following equation.

$$
\frac{d X}{d t}=-k_{d} X
$$

In order to determine the value of $k_{d}$, the growth runs were not stopped, rather the measurement of cell density was continued further for another 5 to 6 days even after the complete consumption of phenol and wattle. Initial substrate concentrations were $250 \mathrm{mgl}^{-1}$ for phenol and wattle, respectively. The data of the decay region were plotted as $\log _{\mathrm{e}}(\mathrm{OD})$ versus time (Figs. 6 and 7). The negative slope gives decay rate coefficient. The values of the decay rate coefficients obtained are 0.0069 and $0.0082 \mathrm{~h}^{-1}$ for phenol and wattle, respectively. These values are in agreement with the earlier studies by Kumar et al. (2005), where the decay coefficient value for phenol and catechol have been reported to be 0.0056 and $0.0067 \mathrm{~h}^{-1}$. The decay coefficient is an important parameter that affects the growth kinetics. Higher the decay coefficient, the wash-out condition would occur at lower dilution rates. The observations from the experiment implies that a reactor treating wastewater containing mainly wattle should be operated at lesser dilution than when treating influent containing mainly phenol.

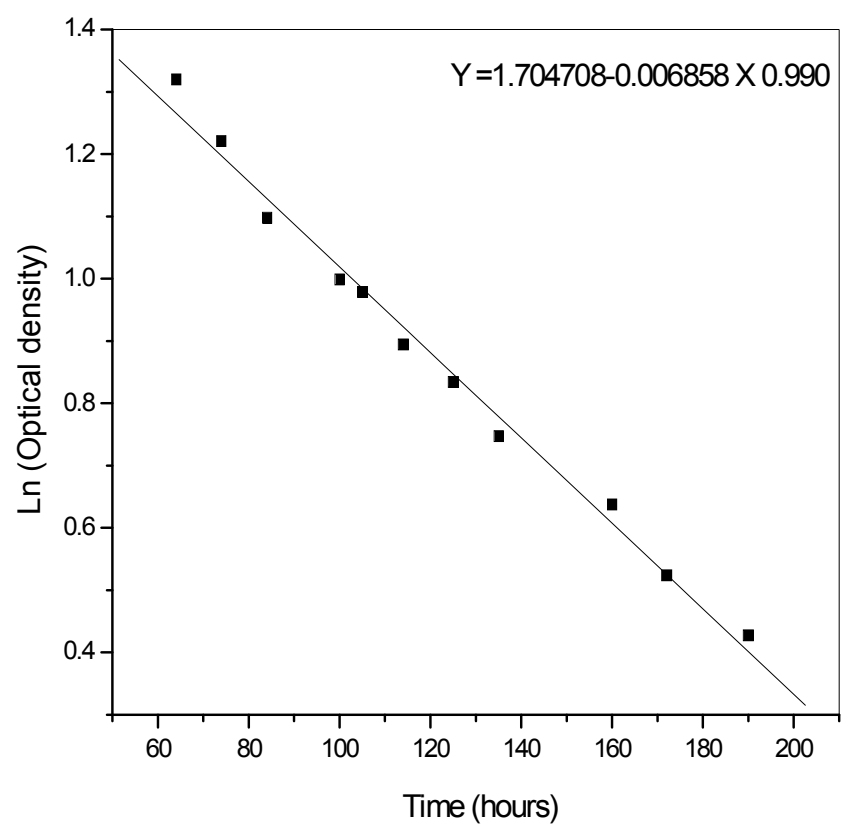

Figure 6. Evaluation of decay coefficient $K_{d}$ for the growth of the mixed consortia containing $P$. aeruginosa and $B$. subtilis on phenol using decay phase growth experimental data 


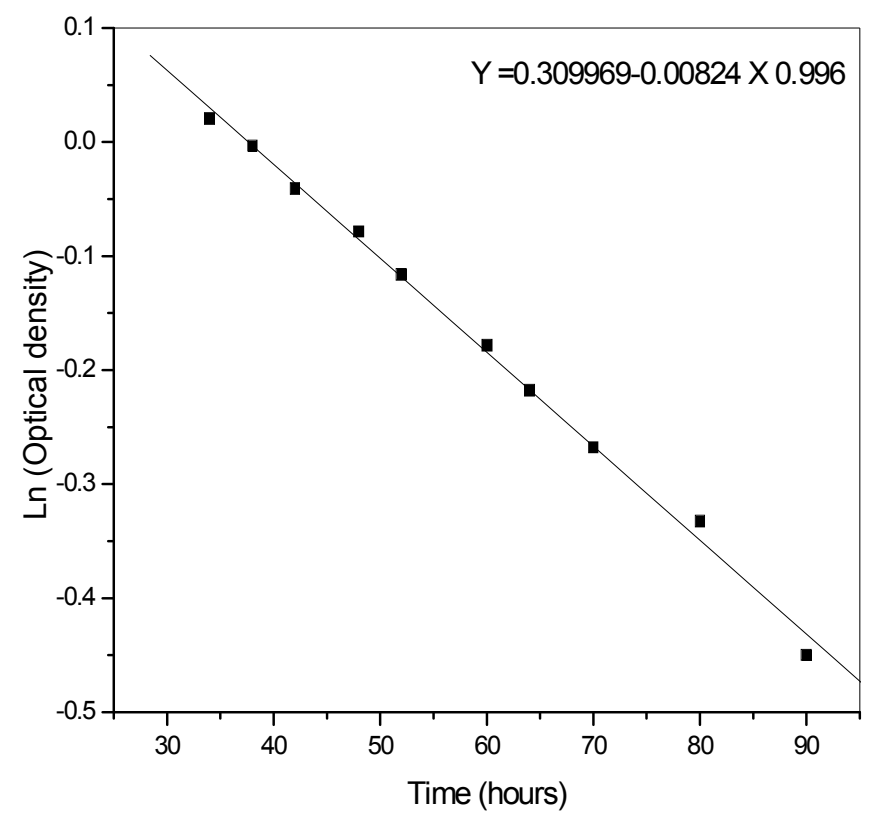

Figure 7. Evaluation of decay coefficient $K_{d}$ for the growth of the mixed consortia containing $P$. aeruginosa and B. subtilis on wattle using decay phase growth experimental data

\section{Conclusions}

The degradation of phenol and a polyphenolic compound by the mixed culture containing Pseudomonas aeruginosa and Bacillus subtilis have been demonstrated. The favourable $\mathrm{pH}$ and temperature for the growth of bacteria has been established to be $7.0 \pm 0.5$ and $37 \pm 2^{\circ} \mathrm{C}$, respectively. The mixed culture was able to grow even at an initial concentration of $500 \mathrm{mgl}^{-1}$ of phenol and wattle. The time taken for the complete degradation of $250 \mathrm{mgl}^{-1}$ of phenol and wattle were estimated to be around 36 and 48 hours, respectively. According to growth kinetics calculation, the degradation of phenol and wattle has been well explained by the Haldane's inhibitory growth model. The growth kinetic parameters such as the maximum specific growth rate, the inhibitory and the half saturation coefficients for the degradation of phenol and wattle have been determined and found to be in accordance with the available literature values. The decay coefficient for the degradation of phenol and wattle has also been calculated from the decay phase of the growth of bacteria. The decay coefficient for the degradation of wattle was high, inferring that while treating the wastewater containing wattle, the reactor should be operated at lesser dilution.

Acknowledgements. The authors thank CSIR for funding under the Supra-institutional Project - S\&T Revolution in Leather with a Green Touch (STRAIT) (CSIR-CLRI communication no.:1068)"

\section{REFERENCES}

[1] Dean-Ross, D., Rahimi, M. (1995): Toxicity of phenolic compounds to environment. Environment Contamination Toxicology 55:245-250. 
[2] Ahmed, S., Hameed, A. (2001): Biotreatment of phenolic wastes from typical pharmaceutical plant. - In: Industrial and Environmental Biotechnology, Horizon Scientific Press, Pakistan, pp. 11-18.

[3] Ojumu, T.V., Bello, O.O., Sonibare, J.A., Solomon, B.O. (2005): Evaluation of microbial systems for bioremediation of petroleum refineries in Nigeria. - African Journal of Biotechnology 4:31-35.

[4] Baradarajan, A., Vijayaraghavan, S., Srinivasaraghavan, T., Musti, S., Kar, S., Swaminathan, S. (1995): Biodegradation of phenol by Arthrobacter and modelling of kinetics. - Bioprocess Engineering 12:227-229.

[5] Zache, G., Rehm, H.J. (1988): Degradation of phenol by co-immobilized entrapped mixed culture. - Applied Microbiology Biotechnology 30:426-432.

[6] Clesceri, L.S., Grenberg, A.E., Eaton, A.D. (1998): Standard methods for the examination of water and waste water, $20^{\text {th }}$ ed. American Public Health Association, USA, pp. 574585.

[7] Cappuccino, J.G., Sherman, N. (1996): Microbiology: A laboratory manual, $4^{\text {th }}$ edition, Addison Wesley Publications.

[8] Livingston, A.G., Chase, H.A. (1989): Modeling phenol degradation in a fluidized bed bioreactor. - AIChE Journal 35:1980-1992.

[9] Edwards, V.H. (1970): The influence of high substrate concentrations on microbial kinetics. -Biotechnology Bioengineering 12:679-712.

[10] Pawlowsky, U., Howell, J.A. (1973): Mixed culture biooxidation of phenol I. Determination of kinetic parameters. - Biotechnology Bioengineering 15:889-896.

[11] Kumar, A., Kumar, S., Kumar, S. (2005): Biodegradation kinetics of phenol and catechol using Pseudomonas putida MTCC 1194. - Biochemical Engineering Journal 22:151-159.

[12] Kumaran, P., Paruchuri, Y.L. (1997): Kinetics of phenol biotransformation. - Water Research 31:11-22. 